\title{
Wittgenstein: incertidumbre instintiva y diversidad conceptual
}

\author{
Carolina Scotto \\ Universidad Nacional de Córdoba/CONICET
}

\begin{abstract}
Resumen: Importantes teorias acerca de la atribución de contenidos mentales y/o de significados lingüísticos proponen una caracterización teoricista acerca de la comprensión mental y lingüística. Entre sus efectos, no pueden dar cuenta de casos de genuina diversidad conceptual: las expresiones exóticas y sus repertorios conceptuales tienen que ser re-descritas por medio de una teoria, expuesta en nuestro propio repertorio conceptual, que elimine esa diversidad. Wittgenstein, por su parte, ha argumentado que la comprensión de la conducta lingüística y no lingüística de otras criaturas se asienta en modalidades primitivas de comprensión reciproca, basadas a su vez en concordancias de orden práctico. Consecuentemente, ha caracterizado nuestra relación con comportamientos radicalmente extraños como una forma de "incertidumbre instintiva". En este trabajo intentaré mostrar cómo es posible sobre estas bases disolver problemas escépticos y evitar soluciones artificiosas acerca de otras "formas de vida", reconociendo que la genuina diversidad conceptual es posible.
\end{abstract}

Palabras clave: Wittgenstein; atribución intencional y semántica; diversidad conceptual; incertidumbre instintiva

Abstract: "Wittgenstein: Instinctive uncertainty and conceptual diversity".
Important theories about the attribution of mental contents and/or linguistic
meanings propose a theoretical characterization about mental and linguistic
understanding. As one of the consequences of this, they cannot account for
instances of genuine conceptual diversity: the exotic expressions and their con-
ceptual repertoires must be re-describe by means of a theory, articulated in our
conceptual repertoire, that eliminates that diversity. Wittgenstein, on the other
side, has argued that understanding of the linguistic and non linguistic behavior
of other creatures is based on primitive ways of reciprocal understanding, settled
on practical agreements. Consequently, he has characterized our relationship
towards radically estrange behaviors as a form of "instinctive uncertainty". On
these bases, I will attempt to show how it is possible to dissolve skeptical problems
and elude contrived solutions about other "forms of life", recognizing that genuine
conceptual diversity is possible.

Keywords: Wittgenstein; semantic and intentional attribution; conceptual diversity; instinctive uncertainty 
La comprensión: los enfoques teoricistas

Importantes teorías de la atribución de contenidos mentales y de significados lingüísticos, independientemente de sus profundas divergencias en una variedad de aspectos, proponen una caracterización teoricista acerca de la comprensión mental y lingüística, esto es, alguna variante de la "teoria"-dela-teoría. Según este enfoque, la "teoria" es el resultado de la observación, la recolección de evidencia, la formulación de hipótesis, la realización de inferencias, la contrastación empírica. La teoría en cuestión es aquella que permite explicar cómo un hablante, en primer lugar, puede asignar significados a sus expresiones lingüísticas, puesto que los hace capaces de identificar las condiciones bajo las cuales esas asignaciones están justificadas. Del mismo modo, dicha teoría es la que permite explicar cómo un individuo forma creencias, tiene deseos, elabora intenciones, etcétera. El mismo papel desempeña la teoría para un interlocutor, para un traductor de una lengua desconocida o para un intérprete, radical o no, respecto de la acción intencional ajena.

Hay que señalar que los enfoques a los que puede atribuirse esta caracterización del problema admiten que los presupuestos, procesos y logros teóricos en cuestión son de un tipo sui generis, es decir, mayormente implícitos, tácitos, inconscientes e incluso parcialmente innatos. Según ciertas teorias, operan a un nivel personal, pero en otras se complementan con procesos que ocurren a un nivel sub-personal. Los enfoques en los que estamos pensando difieren en otros aspectos respecto de su mayor o menor semejanza con las teorias científicas.

Otro rasgo común a estos enfoques es que caracterizan el problema de explicar cómo pasar de evidencia no semántica (ruidos o marcas) o no intencional (movimientos corporales) a contenidos semánticos e intencionales; dicho de otra forma, enfrentan el problema de explicar a partir de datos o evidencia pre-semántica o pre-intencional la construcción de una teoria que pueda interpretar esos datos como semánticos o intencionales. Podría pensarse que este es un rasgo derivado de la pretensión de ofrecer una explicación no circular del problema, es decir, que no presuponga las nociones que se intentan explicar. En efecto, se describen los datos en los que se asienta el significado en un idioma naturalista (de reacciones o disposiciones a la conducta, de estimulación de las superficies sensoriales), pero al hacerlo convierten al problema en un misterio 
$\mathrm{y}$ a la solución en un salto al vacío ${ }^{1}$. Pues bien, este rasgo nada inocente y el modo a través del cual es resuelto por estas teorias serán esenciales para identificar el problema del que queremos ocuparnos.

Esta reconstrucción muy general se aplica, mutatis mutandis, a la teoría conductista del significado (y la traducción) de Quine ${ }^{2}$, a la teoría intencionalista de la interpretación (radical o no) de Davidson³ , a la hipótesis del "lenguaje del pensamiento" de Fodor, y por último, a la teoría del aprendizaje, la comprensión y la actuación lingüística de Chomsky. En este trabajo, sigo la interpretación de Dorit Bar-On de este supuesto común a las teorias mencionadas ${ }^{4}$. Para estas teorias, asignar o atribuir significado a las emisiones de un lenguaje, sea el propio o uno extraño, depende de elaborar (o desarrollar) y luego aplicar una correcta teoría del significado o la traducción; o más ampliamente, atribuir

\footnotetext{
1 A este misterio se ha referido Davidson como un "problema insuperable" al hablar de "la dificultad de describir la emergencia de los fenómenos mentales... Tanto en la evolución del pensamiento en la historia de la humanidad como en la evolución del pensamiento de un individuo hay un estadio en el que no hay pensamiento y que está seguido de un estadio en el que sí lo hay. Describir la emergencia del pensamiento sería describir el proceso que conduce del primero al segundo de estos estadios. Carecemos de un vocabulario satisfactorio para describir los estadios intermedios... Quizás haya un problema insuperable en dar una descripción plena de la emergencia del pensamiento. Doy gracias de no estar en el campo de la psicología del desarrollo" ("La aparición del pensamiento", en: Subjetivo, Intersubjetivo, Objetivo, Madrid: Cátedra, 1997, pp. 176-190, pp. 181-182). Curiosamente, esto es lo que Davidson intenta hacer al dar cuenta de las precondiciones del pensamiento como una relación de triangulación entre dos personas y una entidad objetiva externa. Pero insiste en caracterizar esta interacción en términos pre-semánticos y pre-intencionales, es decir, como una "situación pre-cognitiva" (ibid., p. 182) o de "interacción social primitiva" (ibid., p. 185).

2 Cf. Quine, W.O., Palabra y Objeto, Barcelona: Herder, 2001. Para atribuir esta perspectiva a Quine sería necesario dejar a salvo su reduccionismo conductista, esto es, su anti-intensionalismo radical. La noción teórica de "significado estimulativo" con la que se reemplaza la noción intensional de significado es, sin embargo, una noción semántica construida a partir de evidencia conductual pre-semánticamente caracterizada.

3 Cf. Davidson, D., "De la idea misma de un esquema conceptual", en: De la Verdad y de la Interpretación, Barcelona: Gedisa, 1974/1990, pp. 189-203. Davidson nos dice que “...la capacidad de una persona para interpretar o hablar con otra persona consiste en la capacidad que le permite construir una teoría correcta, es decir, convergente y momentánea para las transacciones lingüisticas con esa persona" (idem, "A Nice Derangement of Epitaphs", en: Davidson, D., Truth, Language, and History, Oxford: Clarendon Press, 1986/2005, pp. 89-107, p. 106). Dicha teoria consiste más precisamente en una "teoría previa" del intérprete respecto de cómo interpretar a un hablante, y una "teoria al paso" (o "momentánea"), respecto como interpretar efectivamente una emisión (ibid., p. 101) Respecto a Fodor y Chomsky, cf. Fodor, J.A., The Language of Thought, Cambridge, Mass.: Harvard University Press, 1975 y Chomsky, N., Reflections on Language, Nueva York: Pantheon, 1975.

4 Bar-On, D., "Semantic Eliminativism, and the "Theory"-theory of Linguistic Meaning", en: Canadian Journal of Philosophy, Supplementary v. XXX (2004), pp. 159-198; idem., "Language, Concepts and Culture: Between Pluralism and Relativism”, en: Facta Philosophica, 6 (2004b), pp. 183-221. La autora discute expresamente la relación entre dicho supuesto y el rechazo del relativismo conceptual y cultural, aunque solo hace una referencia ocasional, en el segundo de los trabajos mencionados, a las ideas de Wittgenstein.
} 
contenidos semánticos y mentales a la acción intencional, lingüística y no lingüística, depende de elaborar (o desarrollar) y luego aplicar una teoría de la interpretación de la acción intencional y del significado lingüístico. Todos sabemos, por otra parte, que estas teorias divergen enormemente e incluso contrastan en innumerables y significativos aspectos, que dejaremos fuera de consideración por exceder los propósitos del presente trabajo.

Por su parte, en el ámbito de la filosofia de la mente y más tarde, en la psicología cognitiva del desarrollo, ha tenido lugar un debate en torno a dos grandes alternativas, la "teoria"-de-la-teoría (TT) y la(s) teoria(s) de la simulación (ST). Estas teorías han intentado explicar la naturaleza de la comprensión psicológica ordinaria, es decir, de nuestra capacidad para leer mentes ("mindreading”) o entender a otros: sus creencias, deseos, emociones, etcétera. La mayor parte de sus protagonistas han atribuido a la psicología de sentido común (PSC) el formato de una teoria, no solo por su estructura y sus contenidos sino también, concomitantemente, en relación con sus propósitos esenciales. Al supuesto teoricista se agregó otro supuesto: el de la primacía de los propósitos epistémicos de la PSC, esto es, se dio primacía a la explicación y predicción de la conducta de otros mediante la atribución de estados mentales, por sobre otras funciones posibles. Si la PSC es una (clase de) teoría explicativa y predictiva de la conducta mediante la atribución de estados mentales, es necesario a su vez explicar sobre qué bases está edificada y cómo puede funcionar ${ }^{5}$. Aunque el debate en la filosofia contemporánea de la mente fue originado por un temprano y sugerente escrito de Sellars ${ }^{6}$ sobre el desarrollo progresivo (a partir de nuestros hipotéticos ancestros ryleanos) de las capacidades teóricas para mentalizar, la idea fue recuperada en la década de 1970 por distintos autores, especialmente aquellos que se acercaron al problema con una motivación epistemológica, como fue el caso de Churchland ${ }^{7}$, entre otros. Según este último,

\footnotetext{
5 Una sumaria revisión de la literatura sobre el tema apoya claramente esta afirmación general, con la excepción de algunas variantes no teóricas de la Teoría de la Simulación. Me refiero a la versión de Gordon, por ejemplo. También en el marco de ese debate fue necesario esclarecer qué noción de teoría estaba siendo usada. Si sus contenidos se determinan externa o internamente (si consiste en una estructura interna de datos o un sistema de representaciones interno), si es innata (Fodor, J.A., o.c.) o aprendida (Gopnik y Wellman, entre otros), o una mezcla de ambos, en qué medida difiere de la noción de teoría aplicable a las teorias científicas, más específicamente en qué sentido es razonable emplear las nociones de observación, evidencia, hipótesis, inferencia, generalización, etcétera.

6 Sellars, W., "Empirismo y la Filosofia de la Mente", en: Ciencia, Percepción y Realidad, Madrid: Tecnos, 1956/1971, pp. 139-209.

7 Churchland, P., "Eliminative Materialism and Propositional Attitudes", en: Journal of Philosophy, v. LXXVIII, 2 (1981), pp. 67-90.
} 
dado que la PSC es una teoría, debe ser evaluada como cualquier otra por sus méritos epistémicos. La conclusión, como se sabe, recomienda eliminarla a favor de una adecuada, completa, es decir, futura, ciencia de la mente. Esta consecuencia extrema, que también se puede atribuir a Quine acerca de los significados ordinarios, muestra que el eliminativismo es una consecuencia posible solo si se adopta un supuesto teoricista previo ${ }^{8}$.

Ahora bien, en este punto, se hace necesario especificar los diferentes fenómenos abarcados bajo el amplio concepto de comprensión empleado hasta aquí. En los autores citados se habla de comprensión (o de interpretación) minimizando o ignorando, implícita o explícitamente, los rasgos distintivos de una variedad de fenómenos, tales como: aprender el lenguaje materno, aprender un nuevo lenguaje, interpretar el comportamiento lingüístico y no lingüístico de otros en el marco de la propia lengua o cultura e interpretar la conducta, lingüística y no lingüística, de personas de una lengua y/o una cultura desconocida ${ }^{9}$. Sin embargo, no es obvio que estos fenómenos sean asimilables en sus rasgos básicos.

Para apreciar mejor algunos posibles contrastes o diferencias, consideremos la reconstrucción de este último caso, el del traductor o intérprete radical. Como se sabe, en las versiones estándar de Quine y Davidson, el problema no es planteado desde la perspectiva de un intérprete o hablante ordinario, alguien que intenta, con una variedad de propósitos prácticos, interactuar con otros desconocidos, contando solo con sus recursos ordinarios. El traductor o el intérprete se asimila más bien al caso de un teórico, lingüista o antropólogo, con el objetivo intelectual de elaborar y testear, conscientemente y de un modo explicito, sus hipótesis y teorias (o manuales), por lo tanto, alguien cuyos propósitos, en tales contextos, son primariamente epistémicos: un observador neutral con pretensiones científicas más que un potencial participante de una interacción cotidiana. A esta caracterización responde claramente el traductor quineano ${ }^{10}$.

\footnotetext{
8 El eliminativismo semántico de Quine y su conexión con el supuesto teoricista está extensamente desarrollado en el trabajo previamente citado de Bar-On, "Semantic Eliminativism, and the "Theory"-theory of Linguistic Meaning".

9 En la mayor parte de los autores de la tradición analítica, los fenómenos de la comprensión y la atribución intencional se restringen a los seres humanos, un punto en el que Wittgenstein constituye una excepción temprana. Como veremos, también Wittgenstein se aparta de los enfoques mencionados en promover reconstrucciones diferentes de cada uno de estos fenómenos.

${ }^{10}$ H.-J. Glock no cree que sea correcto criticar la reconstrucción hipotética del traductor de Quine como si este realizara una labor puramente externa, puesto que, afirma, interactúa dialógicamente con el nativo, lo interroga, es decir, “... no está en la desesperanzada posición de alguien que intenta aprender un lenguaje con la exclusiva ayuda de grabadores y micrófonos colgando de los árboles" (Glock, H.-J., "On Safari with Wittgenstein, Quine and Davidson”, en: Arrington, R.,
} 
En el caso del intérprete radical davidsoniano, en cambio, parece haber una oscilación entre un intérprete teórico en un sentido modesto (alguien que, con una teoría "previa", intenta comunicarse, elaborando "teorías al paso"11) y otro teórico-teórico (alguien que intenta elaborar una teoría explicativa y predictiva de la acción intencional, adoptando más bien una perspectiva de observador). En cualquier caso, la labor de ambos es reconstruida de un modo teórico. Ahora bien, las moralejas que Quine y Davidson extraen respecto de la perspectiva práctica y familiar en las situaciones domésticas de comprensión ordinaria, explícitamente a partir de tales situaciones exóticas y sus correspondientes perspectivas epistémicas radicales, resultan muy desajustadas, al despreciar las importantes diferencias de perspectivas, medios y propósitos. Asimismo, no reconocer sino diferencias de grado entre tales extremos se evidencia, además, en las escasas o débiles justificaciones que proporcionan para mantener un mismo enfoque (e idénticos efectos) para ambos tipos de fenómenos.

Por otra parte, las concepciones de Quine y Davidson de la traducción y la interpretación, respectivamente, combinan la tesis de la indeterminación semántica o intencional, por una parte, con la tesis de la traducibilidad o interpretabilidad de otro lenguaje, sistema de creencias o cultura, por la otra. Según ambos, un lenguaje extraño es traducible o interpretable en la misma medida y del mismo modo en que es aprendible para quienes lo poseen, esto es, en virtud de que es posible asignar significados a sus enunciados como producto de la evidencia pública disponible ${ }^{12}$. Tanto la concepción quineana como la davidsoniana del significado se asientan en lo que Follesdal ha denominado la "tesis del significado hecho por el hombre" (la tesis "MMM": man-made meaning), según

Glock, H.-J. (eds.), Wittgenstein and Quine, Londres/ Nueva York: Routledge, 1996, pp. 144-172, p. 149). Glock sostiene, en cambio, que Quine caracteriza mal la interacción entre el traductor y el nativo porque insiste en aplicar un "método carente de presuposiciones", esto es, considera a los datos básicos de la teoría como no poseyendo un carácter semántico y normativo ab initio. Glock asienta sobre este punto y otros similares que atribuye a Davidson, su propia crítica, apoyándose justamente, en una interpretación similar a la que se defenderá aquí, de las posiciones de Wittgenstein. Estos puntos serán desarrollados enseguida.

${ }^{11}$ La distinción está formulada en Davidson, D., "A Nice Derangement of Epitaphs".

$288 \quad{ }^{12}$ Bar-On denomina "Requerimiento de Publicidad" al supuesto común a las teorias Quine/ Davidson, definiéndolo así: "La correcta comprensión de las expresiones lingüísticas por parte de sus usuarios debe ser en principio posible. Los significados lingüisticos de las expresiones deben ser públicamente accesibles y descubribles por los aprendices de un lenguaje" (cf. Bar-On, D., "Language, Concepts and Culture: Between Pluralism and Relativism"). Davidson acuerda con esta idea atribuyéndosela a Quine: "Quine revolucionó nuestra comprensión de la comunicación verbal tomando en serio el hecho, suficientemente obvio en sí mismo, que no puede haber más respecto al significado que lo que una persona adecuadamente equipada puede aprender y observar" (Davidson, D., "Meaning, Truth and Evidence", en: Barret, R. y R. Gibson (eds.), Perspectives on Quine, Cambridge: Blackwell, 1990, pp. 68-79, p. 78). 
la cual: "El significado de una expresión lingüística es el producto conjunto de toda la evidencia que ayuda a quienes aprenden y a los usuarios del lenguaje a determinar ese significado"13. Follesdal atribuye a Quine la adhesión a esta concepción evidencial del significado, pero también a Davidson, cambiando en su caso la naturaleza de la evidencia (i.e. la activación de superficies sensoriales y las disposiciones al comportamiento lingüístico por la interacción causal ordinaria con objetos macroscópicos y su relación con actos de emisión lingüística) ${ }^{14}$. En ambos casos, la teoría debe pasar de la evidencia no semántica a la evidencia semántica y esto la convierte, justamente, en una teoría semántica. Ahora bien, si por una parte, la totalidad de la evidencia (empírica y conductual) es toda la evidencia disponible, es también claro, por otra, que dicha evidencia es insuficiente para determinar una asignación precisa o determinada de significados. A partir de aquí la semejanza entre las teorias del significado y las teorias científicas se rompe, porque mientras las primeras exploran una estructura "hecha por el hombre", estas últimas "exploran una estructura que no es de nuestra propia hechura, o al menos no totalmente de nuestra propia hechura..."15. Mientras las teorias científicas pueden postular además hechos inobservables, los que en principio permitirian superar la sub-determinación teórica, la indeterminación semántica, en cambio, es una situación definitiva.

Según las concepciones de Chomsky y de Fodor, por otra parte, una adecuada teoría del lenguaje (de su gramática y sus reglas, e incluso de algunos conceptos o contenidos semánticos), requiere postular la existencia de sucesos internos en la mente/cerebro de los hablantes, más allá de la evidencia empírica proporcionada por la conducta. Acerca de la evidencia conductual nuestras hipótesis tienen el mismo grado de falibilidad que en el caso de otras teorías acerca de otros dominios de la realidad. Así, la indeterminación a la que conduce la sola evidencia conductual es superable por la determinación que aporta la

\footnotetext{
13 Follesdal, D., "Indeterminacy and Mental States", en: Barret, R. y R. Gibson (eds.), Perspectives on Quine, Cambridge: Blackwell, 1990, p. 103.

${ }_{14}$ Davidson explicita la diferencia (y desarrolla sus efectos) al distinguir, según la localización del estímulo, dos teorías: la "teoría del estímulo próximo" -la de Quine- y la "teoría del estímulo distal" -la suya propia- (c $f$. Davidson, D., "Meaning, Truth and Evidence", p. 73). Sostiene, además, que mientras la teoría del estimulo próximo hace depender directamente al significado de la evidencia, la teoría del estímulo distal, como la suya, considera primaria a la verdad más que a la evidencia: "la teoría distal conecta el significado directamente a las condiciones que hacen intersubjetivamente verdaderos o falsos a los enunciados" (ibid., p. 75.) La diferencia es relevante porque, según argumenta Davidson, solo las primeras se ven afectadas por problemas escépticos, ya que "...basar el significado en la evidencia necesariamente lleva a las dificultades de las teorias del estímulo próximo: la verdad es relativizada a los individuos...” (ibid., p. 76).

${ }^{15}$ Follesdal, D., o.c., p. 194.
} 
teoria en la mente de los hablantes y en la mente de los intérpretes. Dejo aquí sin comentar otros importantes rasgos comunes entre las teorias de Chomsky y Fodor y sus afinidades (y contrastes) con las teorias de Quine y Davidson ${ }^{16}$.

El punto que nos interesa es que, a pesar de estas importantes diferencias (indeterminación vs. determinación, entidades públicas y observables vs. entidades teóricas inobservables), en ninguno de ambos tipos de teorias es posible hacer lugar a casos de genuina diversidad conceptual. Las respuestas de Fodor y Chomsky y de Quine y Davidson pueden tomarse como expresiones de "parroquialismo" o de "imperialismo"17, respectivamente, dependientes del común enfoque teoricista antes señalado. Según el parroquialismo, podemos interpretar a otros en forma determinada y clara, porque tenemos la misma teoria; según el imperialismo, podemos interpretar a otros asumiendo indeterminación pero resolviéndola con los recursos no opcionales de nuestra teoría, es decir, de nuestros conceptos, creencias, conocimientos y patrones de racionalidad. Independientemente de sus diferencias, obtenemos o bien los mismos conceptos y creencias o bien nuestros conceptos y creencias, es decir, otra vez, los mismos conceptos y creencias.

Nos ocuparemos ahora de examinar los rasgos de la comprensión en la obra de Wittgenstein, por representar claramente una posición alternativa en la que es posible reconocer el fenómeno de la diversidad conceptual.

\section{La comprensión reciproca}

Wittgenstein se ocupó en diversos lugares del problema filosófico del aprendizaje del lenguaje, argumentando en contra de las explicaciones sobreintelectualizadas de la clase de esfuerzos que supondría para un niño adquirir su primera lengua. Los pasajes dedicados a Agustín con los que comienzan las Investigaciones Filosóficas $(I F)^{18}$ tienen, entre otros, ese propósito crítico.

\footnotetext{
${ }^{16}$ En los trabajos citados de Bar-On puede seguirse una pormenorizada discusión de las semejanzas entre Fodor/Chomsky y Quine/Davidson.

17 Estos pueden considerarse enfoques cognitivos en general. Sin embargo, según cuáles rasgos se 290 deseen considerar, pueden considerarse enfoques más específicos. En general abarcan conceptos, creencias y normas o patrones de racionalidad. Un tratamiento específico e independiente de cada uno de ellos permite reconocer diversas combinaciones posibles, es decir, versiones más fuertes o más débiles de imperialismo y de parroquialismo. La distinción pertenece a Ramberg, B., Donald Davidson's Philosophy of Language: An Introduction, Oxford: Basil Blackwell, 1989, y es aplicada por Bar-On a Davidson y a Fodor, en "Language, Concepts and Culture: Between Pluralism and Relativism", con el sentido que le hemos dado aquí.

18 Wittgenstein, L., Investigaciones Filosóficas, México: Crítica, 1998; en adelante, IF. En adelante, se citarán los textos de Wittgenstein como es usual, indicando las iniciales del título y el número de parágrafo de la obra respectiva, cuando corresponde.
}

ARETÉ Revista de Filosofía, vol. XXVIII, N², 2016 / ISSN 1016-913X 
Wittgenstein criticó a Agustín por haber asimilado al niño que aprende un primer lenguaje con el traductor de un lenguaje extraño. En su conocida crítica al modelo agustiniano del aprendizaje del lenguaje humano, nuestro autor apuntó directamente a sus presupuestos intelectualistas y teoricistas: el niño de Agustín debería ser capaz de pensar y operar como el traductor quineano, porque es “...como si el niño llegara a un país extraño y no entendiera el lenguaje de ese país; esto es, como si ya tuviese un lenguaje, solo que no ese"19. Debería poseer además un sistema interno de representaciones (obviamente innato) con ayuda del cual formularse hipótesis para asignar significados a los sonidos de los adultos, apoyándose en la observación del resto de su conducta. Así es que el niño de Agustín debería ser también previamente competente con algún lenguaje mental, “...como si el niño ya pudiese pensar, solo que no hablar. Y 'pensar' querría decir aquí algo como: hablar consigo mismo"20. La intuición agustiniana acerca de las precondiciones mentales del aprendizaje lingüístico, consistente en poseer ya otro lenguaje mental innato, fue desarrollada, entre otros, por Fodor con su hipótesis de un "lenguaje del pensamiento". Como Quine, también Fodor asimila el aprendizaje del lenguaje por parte del niño a la interpretación adulta del lenguaje de otros: en ambos casos se requiere la traducción de las emisiones al lenguaje mental del intérprete.

El problema del aprendizaje de un lenguaje es descrito, en cambio, como un cierto adiestramiento ${ }^{21}(I F, 5)$ para la adquisición de habilidades o técnicas para el empleo de las herramientas lingüísticas o como "ajustar el mecanismo a un determinado tipo de influencia"22. Wittgenstein cuestionó igualmente los supuestos intelectualistas de la comprensión semántica ordinaria, describiendo los casos de seguimiento de reglas como efectos del adiestramiento en las diversas prácticas normativas comunales, las que presuponen acuerdos

\footnotetext{
19 IF, 32.

20 Ibid.

21 Cf. IF, 5.

22 Así caracteriza el aprendizaje de una lengua particular, en el parágrafo 495 de las Investigaciones Filosóficas. Para un análisis pormenorizado de diversos tópicos acerca de la concepción wittgensteineana de los conceptos, se puede ver el siguiente trabajo: Glock, H.-J., "Wittgenstein on Concepts", en: Ahmed, A., Wittgenstein's Philosophical Investigations: a Critical Guide, Cambridge: Cambridge University Press, 2010, pp. 88-108. En este texto, Glock destaca un aspecto de la concepción wittgensteineana que contrasta claramente con los que llamamos aquí enfoques teoricistas: "El significado de una expresión es simplemente lo que comprenden los hablantes competentes, y lo que ellos le explican a los neófitos. Del mismo modo, lo que los hablantes dicen al usar expresiones es simplemente lo que captan aquellos que entienden sus emisiones. Cualquier explicación que abra las compuertas al escepticismo semántico sencillamente pierde el concepto de significado. Los factores ocultos son ruedas que giran en el vacío, semánticamente hablando" (ibid., p. 99).
} 
en los comportamientos ${ }^{23}$. De un modo análogo, caracterizó las atribuciones psicológicas (la comprensión de "otras mentes") mostrando que, en condiciones normales, no se plantean desafios escépticos y por lo tanto, no se requieren esfuerzos intelectuales de tipo teórico o cuasi-teórico por parte del intérprete (por ejemplo, inferencias a partir del modelo igualmente teórico que brinda el auto-conocimiento), sino solo el seguimiento de las reglas propias de esa práctica conceptual, asentadas en prácticas normativas más amplias y, más básicamente aun, en patrones de comportamiento compartidos, algunos incluso de carácter natural ${ }^{24}$.

Las criticas de Wittgenstein a ciertas interpretaciones filosóficas del aprendizaje del lenguaje por parte del niño, de la comprensión semántica y de la comprensión psicológica ordinarias son suficientemente conocidas, por lo que no las desarrollaré aquí. Solo voy a agregar algunos aspectos positivos de su propia manera de ver estos problemas, que no aparecen en las reconstrucciones clásicas de la posición de Wittgenstein o bien están desdibujados o son, incluso, mal interpretados. Con ello, me refiero a la conexión entre ciertas formas básicas de interacción intencional, su carácter práctico y el tipo de "certidumbre" que las acompaña. De ese modo, será posible abordar

\footnotetext{
${ }^{23}$ Wittgenstein señaló claramente que el uso correcto de las palabras no depende de una previa interpretación de las reglas, independientemente de sus aplicaciones: "hay una captación de una regla que no es una interpretación, sino que se manifiesta, de caso en caso de aplicación, en lo que llamamos "seguir una regla" y en lo que llamamos "contravenirla" (IF, 201). En (IF, 242) se hace explícito este círculo entre los acuerdos en las reglas y los acuerdos en sus aplicaciones: "A la comprensión por medio del lenguaje pertenece no solo una concordancia en las definiciones, sino también (por extraño que esto pueda sonar) una concordancia en los juicios”. De igual modo, "la comunicación lingüística normal" no depende de la posesión de teoría alguna: "Si no desconfio de él, no me preocupo de lo que ocurra en él. (Las palabras y su significado. El significado de las palabras, lo que está detrás de ellas, no me preocupa en la comunicación lingüística normal. Las palabras fluyen, y se lleva a cabo la transición de las palabras a las acciones y de las acciones a las palabras. Nadie piensa, al hacer cálculos aritméticos, en si los hace 'reflexivamente' o 'a la manera de un papagayo' (Frege)" (Wittgenstein, L., Observaciones sobre los Fundamentos de la Psicología, t. II, v. II, México: Crítica, 1997, 603; en adelante, OFP).

24 "Creer que el otro tiene dolor o dudar de que lo tenga son otras tantas formas naturales de conducta en relación con los otros seres humanos. Nuestro lenguaje no es sino un recurso y una extensión de esta conducta. Lo que quiero decir es esto: nuestro juego de lenguaje es una extensión de la conducta más primitiva. (Porque nuestro juego de lenguaje es conducta). $(Z, 545)$ ", (OFP, v. I, 151) referido a Wittgenstein, L., Zettel, México: Crítica, 1985; en adelante, $Z$. Es indiscutible que Wittgenstein asienta el aprendizaje del "juego de lenguaje psicológico" en las reacciones naturales o espontáneas de carácter gestual o pre-verbal. Ahora bien, estas expresiones instintivas o primitivas obedecen a nuestra conformación biológica y, por lo tanto, serian universales. Sin embargo, en otros textos Wittgenstein parece admitir, al menos en la imaginación, que es posible que otros seres humanos expresaran sus sensaciones (dolor, por ejemplo) o sus emociones (alegria, por ejemplo) de formas radicalmente diferentes. Volveremos sobre este punto más adelante.
} 
mejor el problema de la comprensión de lenguajes y conductas extrañas, los casos de diversidad conceptual.

Atribuyo a Wittgenstein la idea de que para reconstruir adecuadamente nuestras formas más sofisticadas de comprensión semántica e intencional, es necesario identificar primero formas de comprensión básica, pre-lingüísticas pero ya intencionales, consistentes en un conjunto de habilidades interactivas, anteriores a la distinción nítida entre un yo psicológico pleno y una mente ajena, y que sobreviven a ella y la acompañan siempre. Estas formas de comprensión reciproca no pueden ser descritas como una comprensión teórica de la mente ${ }^{25}$ de otros sino como una comprensión práctica entre personas ${ }^{26}$. Se trata de habilidades intencionales porque solo pueden identificarse por sus contenidos, en una red de otros contenidos relativos a otras personas, y solo pueden comprenderse en la medida en que esos contenidos sean adecuadamente identificados, desde el inicio, en un nivel intencional. Decimos que se trata de modalidades prácticas de entendimiento porque se expresan en acciones acordes, o mejor, en la coordinación o complementariedad de las reacciones entre personas, de acuerdo a propósitos compartidos o en conflicto, pero sensibles a aquellos contenidos. Cuando todo anda bien, la comprensión no lingüística de gestos o expresiones de estados intencionales (especialmente de sensaciones, emociones y acciones orientadas a fines básicos) consisten para Wittgenstein en una clase de "acuerdo espontáneo" o "simpatía espontánea"27. En efecto, las reacciones de contagio emocional, consuelo, compasión, auxilio y similares, así como la identificación de comportamientos sinceros, dubitativos, distraídos, confiables, y sus contrapartes ${ }^{28}$, no pertenecen a nuestras reacciones o disposiciones bioló-

\footnotetext{
25 "Dices que atiendes a alguien que se queja, porque la experiencia te ha enseñado que tú mismo te quejas al sentir tal y cual cosa. Pero, en vista de que no extraes una conclusión de este tipo, podemos dejar de lado esta justificación por analogia. (Z, 537)". (OFP, v. I, 719)

${ }^{26}$ En este sentido, es enteramente correcta, en mi opinión, la interpretación de Dennett de la visión de Wittgenstein (y de Ryle) como habiendo elaborado una forma específica de reflexión psicológica, que él denominó "psicología del nivel personal". La distinción de los niveles personal y sub-personal, hoy ampliamente extendida en los debates teóricos y meta-teóricos en filosofia de la mente y de las ciencias cognitivas, fue propuesta, entre otras buenas razones, para evitar algunas características confusiones que surgen de no distinguir en qué nivel se plantean las preguntas (y las respuestas). Su aplicación a la interpretación de las investigaciones wittgensteineanas sobre los fenómenos de la atribución intencional y semántica como fenómenos pertenecientes al nivel personal hubiera evitado (y debería evitar) errores y confusiones en la valoración de sus presupuestos y efectos. (Cf. Dennett, D., Contenido y Conciencia, Barcelona: Gedisa, 1969/1996).

27 "El acuerdo espontáneo, la simpatía espontánea, es lo que, de cualquier modo, resulta esencial para nosotros" (OFP, v. I, 699).

${ }_{28}$ Muchos fragmentos en las $I F$, en $Z$ y en las $O F P$ se pueden comprender a la luz de estas modalidades de comprensión práctica entre personas o comprensión recíproca.
} 
gicas. No estamos hablando de estornudos, picazones, bostezos, sudoraciones, etcétera, sino de un primer grado de nuestra comprensión intencional humana, la específicamente social. En este nivel ya es posible discernir contenidos intencionales y patrones normativos mínimos. En efecto, no vemos primero objetos físicos redondeados que en algún momento preciso y por mediación de una teoria, pasarán a ser rostros conocidos y amigables ${ }^{29}$. De igual modo, no oímos primero ruidos vocales que en algún momento preciso se convertirán en palabras significativas. Por esta razón, no tiene sentido intentar trazar un limite nítido que separe los brutos comportamientos animales de las plenas acciones normativas: se trata de un nivel al que ingresamos gradualmente ${ }^{30} \mathrm{y}$ sin realizar esfuerzos teóricos, al mismo tiempo que nos vamos convirtiendo gradualmente en personas ${ }^{31}$. Diversos propósitos prácticos constituyen la red de interacciones intencionales entre las personas y distintos fenómenos psicológicos están involucrados en ellas: sensaciones, emociones, estados de ánimo, intereses, deseos. Nuestros esfuerzos epistémicos más básicos están enlazados con esa diversidad de fenómenos y con aquellos propósitos prácticos.

Ahora bien, deciamos que deben identificarse primero porque sin ellos no podrian a su vez explicarse los aspectos básicos del aprendizaje y de la comunicación lingüística ordinaria, no solo como sus pre-condiciones necesarias (las condiciones davidsonianas de la triangulación ${ }^{32}$ ) para la adquisición de habilidades plenamente conceptuales y normativas, sino también como condiciones presentes en el ejercicio de las habilidades intencionales más complejas, sin las cuales la mayoría de ellas no podrían funcionar. Wittgenstein no asimila estos casos al que plantea la comprensión de una "forma de vida" diferente porque

\footnotetext{
${ }^{29}$ En diversos fragmentos de las $I F, Z$ y $O F P$ se hace explícito el carácter intencional básico y no inferido de los casos más simples de comprensión. Por ejemplo: "Uno ve la emoción" -¿En oposición a qué cosa? -No vemos las muecas de una persona e inferimos que siente alegria, aflicción, aburrimiento. Describimos directamente su rostro como triste, radiante, aburrido, aunque no seamos capaces de dar ninguna otra descripción de sus rasgos. -La tristeza se personifica en el rostro, quisiera uno decir. Esto resulta esencial para lo que llamamos 'emoción"' (OFP, v. II, 570). “¿Acaso no puedo decir: el grito, la risa, están llenos de significado?” (IF, 543).

${ }_{30}$ "....uno no dice de un lactante que espera... pero sí lo dice de una persona ya mayor. -Bueno,

294 la vida cotidiana se convierte cotidianamente en algo en lo que hay un espacio para la esperanza. (Z 469a)" (OFP, v. I, 15).

${ }^{31}$ Glock en "On Safari with Wittgenstein, Quine and Davidson”, señala sobre todo en este punto el contraste entre las teorias Quine/Davidson y la perspectiva de Wittgenstein: "Lo que hace posible la comprensión e interpretación ordinaria no es evidencia más allá de la conducta humana, como los oponentes mentalistas de Quine y Davidson han sostenido. Más bien es el hecho de que para quienes participan tal comportamiento es ab initio infundido con significado e intenciones. Lo que encontramos no son meros patrones sonoros y movimientos corporales, sino comportamiento guiado por reglas" (p. 164), cursivas de la autora.

32 Cf. Davidson, D., "La aparición del pensamiento".
} 
justamente compartir una "forma de vida" es la precondición más básica para que puedan darse estas formas de comprensión inmediata, lingüística y no lingüística. A su vez, concordar en una "forma de vida" no es compartir creencias, opiniones o visiones del mundo ${ }^{33}$. Otra vez, lo que propone es una visión no intelectualizada de los acuerdos o concordancias, en este caso, de aquellos que permiten la comprensión.

\section{Certidumbres prácticas}

En Sobre la Certeza, Wittgenstein analizó el significado y papel de los "juegos de lenguaje epistémicos", sobre todo, la aplicación de predicados epistémicos como "sé", "dudo", "estoy seguro", etcétera. A las visiones filosóficas clásicas sobre el alcance de estos predicados, opuso una caracterización práctica de los acuerdos, reubicando drásticamente el papel de todos los predicados epistémicos ${ }^{34}$ y el de las certezas o certidumbres que expresan. El objetivo de Wittgenstein sigue siendo desmontar los intentos sobre-intelectualizados de explicar los fenómenos corrientes de nuestra vida, en este caso, los intentos fundacionalistas de apoyar nuestras creencias falibles en certezas inconmovibles, no menos que desmontar supuestos desafios escépticos, mostrando su común dependencia de una concepción sobre-intelectualizada de la duda y de la suposición de que esta solo puede ser despejada apoyando nuestras creencias en fundamentos teóricos indubitables. Pero las certidumbres prácticas no conforman una visión del mundo construida o aprendida, sino un "marco de referencia" o "trasfondo" (Hintergrund) ${ }^{35}$ contra el cual tienen sentido nuestras creencias, dudas y conocimientos. Por esta razón, tienen el mismo carácter que aquellas formas básicas y compartidas de comprensión intencional reciproca. $\mathrm{Si}$ este es su papel y su importancia y si, a su vez, no tuvieran carácter universal,

\footnotetext{
33 “ ¿Dices, pues, que la concordancia de los hombres decide lo que es verdadero y lo que es falso?" Su respuesta es: "Verdadero es lo que los hombres dicen; y los hombres concuerdan en el lenguaje. Esta no es una concordancia de opiniones sino de formas de vida” $(I F, 241)$.

${ }^{34}$ Wittgenstein interpreta "sé" o "dudo" como predicados mucho más especializados, es decir, de un empleo más acotado y local que el que le asignaron las visiones filosóficas que está criticando. En el contexto epistémico, esas visiones filosóficas (solidarias entre sî) son básicamente el fundacionalismo y el escepticismo.

${ }^{35}$ Wittgenstein emplea ambos conceptos con igual significado. Por ejemplo el de trasfondo, en (OFP, v. II, 625): "El trasfondo es el engranaje de la vida. Y nuestro concepto designa algo en ese mecanismo"; o "¿Cómo podría describirse la conducta humana? Con toda seguridad solamente señalando las conductas de una diversidad de seres humanos, tal y como éstas se entrecruzan. El trasfondo sobre el que vemos un acción y que determina nuestros juicios, nuestros conceptos y reacciones no es lo que alguien hace ahora, sino el barullo (de las acciones de los seres humanos) en su totalidad" (OFP, v. II, 629).
} 
entonces son posibles certidumbres, comportamientos y conceptos también diferentes. En efecto, es al menos posible imaginar otras "formas de vida".

Ahora bien, Wittgenstein no solo explora la posibilidad de "formas de vida" diferentes sino que las considera un hecho, tanto en cada una de las distintas especies animales como en el interior de la especie humana ${ }^{36}$. La opinión estándar, tanto vulgar como filosófica, es que en el caso de los animales, las diferencias entre sus "formas de vida" y las nuestras son cualitativas, esto es, nuestros puntos de contacto solo se explican por nuestra mayor convivencia con ciertas especies sumada a una tendencia antropomorfizante que nos permite mantener con ellos un trato fluido o próximo pero literalmente inapropiado ${ }^{37}$. Para Wittgenstein, en cambio, tendriamos que distinguir gradualmente entre aquellas especies más próximas, biológica y culturalmente (como monos y perros, por ejemplo), y aquellas más distantes (como leones, peces o insectos). Las diferencias entre nosotros y el primer tipo de animales serian más bien de grados, en lo que respecta a cierto tipo de comportamientos (y a las competencias cognitivas que suponen), pero son, en el segundo, dramáticamente cualitativas (¿'es por eso que resultan extrañas las atribuciones antropomórficas a ellas?). Estas diferencias se expresan, concomitantemente, en mayores o menores certidumbres (e incertidumbres) prácticas frente a lo que puedan significar sus comportamientos en relación con sus análogos en nuestro caso. En este contexto, Wittgenstein introduce su noción de "incertidumbre instintiva" o "práctica" y la contrasta con una duda en el pensamiento, aplicándola, por ejemplo, a la incertidumbre acerca de si una mosca o una araña siente o no dolor:

"Piensa en la incertidumbre acerca de si los animales, en particular, los animales de las especies menores, como las moscas sienten dolor. La incertidumbre acerca de si una mosca siente dolor es de carácter filosófico. Pero ¿no podría también ser una incertidumbre instintiva? ¿Y cómo se haría ver esto? ¿No existe, de hecho, una incertidumbre en nuestra conducta en relación con los animales? Uno no sabe si él es cruel o no"38.

\footnotetext{
${ }^{36} \mathrm{El}$ concepto de "forma de vida" es utilizado en la literatura wittgensteineana sobre todo en su aplicación a seres humanos, y es correcto en la medida en que Wittgenstein lo utilizó sobre todo en referencia a ellos, pero está claro que es aplicable también a animales.

${ }^{37}$ Las raíces y alcances de la práctica antropomórfica de atribuir propiedades o características humanas a otras especies merece una discusión independiente, tanto como un fenómeno propio de la psicología ordinaria (el nivel en el que Wittgenstein se interesó por ella), cuanto desde el punto de vista de su utilidad heurística o justificación empírica en etología cognitiva.

${ }_{38}$ OFP, v. II, 659.
}

ARETÉ Revista de Filosofía, vol. XXVIII, N², 2016 / ISSN 1016-913X 
"Porque existe una incertidumbre en nuestra conducta que no se encuentra basada en una incertidumbre en el pensamiento" 39.

"Pero si pongo en duda que una araña sienta dolor, esto no se debe a que yo sepa qué tengo que esperar" 40 . Estos ejemplos son especialmente pertinentes, porque es bastante obvio que las semejanzas entre los comportamientos de animales como los insectos y los nuestros son en extremo débiles, si es que encontramos algunas. Pero dado que nos interesa aquí abordar la cuestión de las divergencias conceptuales, y que no podemos dar por supuesto que algunos animales posean habilidades conceptuales ${ }^{41}$, vamos a considerar ahora el caso de formas humanas de vida diferentes y de repertorios conceptuales también diferentes. De hecho, como veremos, Wittgenstein caracteriza de igual modo la incertidumbre que producen formas de comportamiento extrañas, oponiendo la incertidumbre práctica, primitiva, instintiva o real a la incertidumbre filosófica, del pensamiento o incluso ficticia ${ }^{42}$.

\section{Incertidumbre instintiva y diversidad conceptual}

La posición de Wittgenstein respecto de los marcos o esquemas conceptuales es escurridiza: por una parte, pareciera que a cada forma de vida le corresponde uno (o varios) esquemas conceptuales propios, pero, por la otra, parece contrastar el concepto de forma humana de vida, con sus marcos conceptuales, al de formas de vida de seres no humanos, animales por ejemplo. Es posible justificar esta distinción atribuyendo a Wittgenstein la creencia que hay algo así como un repertorio común de formas humanas de comportamiento, dependientes de nuestra naturaleza biológica o de nuestra "historia natural". A favor de esa opinión, se puede citar, entre otros, el fragmento 206 de $I F$, donde se puede leer: "El modo de actuar humano común es el sistema de referencia por medio del cual interpretamos un lenguaje extraño"43. Como el mismo texto sugiere, estas formas de comportamiento comunes a los seres humanos, harian posible la traducción de una lengua desconocida.

\footnotetext{
39 Ibid., 660.

40 Ibid., 667 y Z, 564. Cursivas de la autora.

41 Son crecientes los estudios sobre la cognición animal que examinan en qué medida pueden atribuirse competencias conceptuales a ciertas especies animales, pero la cuestión es todavía materia de controversias. En mi opinión, es posible atribuir a Wittgenstein la idea que algunas competencias conceptuales básicas pueden atribuirse justificadamente a ciertas especies animales.

42 Cf. OFP, v. II, 558, 561, 644, 660 y 662.

${ }^{43}$ Cursivas de la autora.
} 
No obstante, esta idea no refleja apropiadamente otras observaciones de Wittgenstein acerca de la existencia (y la posibilidad) de una enorme variabilidad e imprevisibilidad en los comportamientos humanos ${ }^{44}$. Refiriéndose a la "naturaleza humana" 45 como asiento para la formación de conceptos, Wittgenstein concluye que "la naturaleza compleja y la variedad de los acontecimientos humanos constituyen un fundamento natural de esta construcción conceptual..."46, de donde se puede inferir que nuestros conceptos presentan una enorme variedad y complejidad. Esta observación es mejor comprendida si se toma en cuenta además el papel que para Wittgenstein tienen las instituciones humanas, la educación y las costumbres. Sobre los rasgos comunes de raiz "instintiva" se sobreponen entonces otros comportamientos y se constituyen nuevos conceptos, como capas menos compartidas e incluso muy diferentes, que son el fruto del desarrollo cultural de cada comunidad humana particular. Por esta razón, "una educación completamente diferente de la nuestra podría ser también el fundamento de conceptos completamente diferentes de los que tenemos $(Z, 387) " 47$.

Por su parte, aunque se sugiere que todos los conceptos son herramientas construidas por los seres humanos, de donde se sigue que no habría conceptos innatos y, por lo tanto, comunes a todos los seres humanos, los esquemas o marcos conceptuales construidos de modo contingente, aun siendo potencialmente variables, llegan a constituir en nosotros una "segunda naturaleza", determinando los pensamientos conformados por ellos ${ }^{48}$. Este modo de entender el problema elimina la aparente paradoja entre el carácter contingente de nuestras formaciones conceptuales y su carácter determinante o necesario para nuestros juicios y acciones ${ }^{49}$.

Además, Wittgenstein imagina en muchos lugares la posibilidad de esquemas conceptuales diferentes ${ }^{50}$. Es curioso y digno de destacar que los ejemplos

44 OFP, v. II, 663, 664; ver también 627 y 636.

45 Ibid., 607 y 608.

46 Ibid, 614.

47 Ibid., 707.

48 "Estamos habituados a una clasificación particular de las cosas. Con el lenguaje o los lenguajes, esa clasificación se ha convertido en una segunda naturaleza para nosotros." (OFP, v. II, 678), cursivas de la autora.

49 Refiriéndose a nuestros conceptos: "Estos son los rieles fijos por los que transitan todos nuestros pensamientos y, en consecuencia, conforme a los cuales se desenvuelven nuestros juicios y acciones" $(Z, 375)$.

50 Por ejemplo, en OFP, v. I, 700, donde hace referencia a seres humanos que en diversos aspectos fueran como nosotros, pero en otros resultaran completamente incomprensibles. "O: Nos causaria una profunda y extraña impresión el toparnos con personas que solamente conozcan la música 
de conceptos que podrian ser diferentes no solo incluyen a aquellos más obviamente relativos a la educación o a las instituciones de cada comunidad ${ }^{51}$. Los conceptos psicológicos asociados a las reacciones naturales o comportamientos expresivos espontáneos, tales como el de dolor, parecen ser relativos también a las "formas de vida", y estas podrian ser diferentes de las nuestras. No está claro, sin embargo, si estos ejercicios etnográficos puramente especulativos frecuentes en sus escritos se proponen algo más que generar la distancia necesaria para contemplar el carácter de "nuestros" $\operatorname{conceptos}^{52}$. Por ejemplo, se puede imaginar una comunidad que tuviera dos y no un concepto de dolor, uno para heridas que suscitan cuidados, compasión, etcétera, y el otro para algunos dolores que suscitan burlas, de modo que las semejanzas en los comportamientos que para nosotros justifican solo un concepto, nos obligan a preguntarnos: “¿Pero realmente no notan la semejanza? - ¿Disponemos de un concepto siempre que hay semejanza? La pregunta aquí sería (más bien), ¿Resulta importante para esta gente esa semejanza? ¿Tiene que serlo? $(Z, 380)$ "53 54.

En vista de todos los puntos señalados hasta aquí, se puede afirmar que mientras la comprensión psicológica entre personas de una misma "forma de vida" se expresa en certidumbres prácticas (aunque sin excluir del todo casos de incertidumbre, según el intérprete de que se trate, o dudas locales en ocasiones particulares, acordes con el tipo de juego de que se trata55), en el caso de

de los relojes. Tal vez esperariamos gestos de un tipo desconocido para nosotros ante los que no sabriamos cómo reaccionar" (OFP, v. I, 696). Se recomienda ver también OFP, v. I, 149.

${ }^{51}$ La existencia de una diversidad conceptual en ciertas regiones conceptuales específicas, lo que a su vez explicaría el uso del concepto de forma de vida en plural, no justifica por sí sola la interpretación plenamente culturalista de esa diversidad, puesto que otros conceptos podrian ser comunes o al menos muy similares en el repertorio conceptual humano. Esta es, después de todo, una cuestión empírica, y como tal no puede resolverse de manera especulativa.

${ }^{52}$ Refiriéndose a las prácticas rituales que describe Frazer, Wittgenstein afirma que "uno podría inventar perfectamente prácticas primitivas y sería una casualidad que no se encontrasen realmente en alguna parte" (Wittgenstein, L., "Observaciones sobre La Rama Dorada de Frazer", en: Ocasiones filosóficas. 1912-1951, Madrid: Cátedra, pp. 144-163, p. 148).

${ }^{53}$ OFP, v. II, 638.

${ }^{54}$ Wittgenstein imagina a otra tribu “...que fuera educada para que se abstuviera de manifestar una expresión de sentimientos de cualquier tipo. Tales personas lo consideran algo infantil que tiene que suprimirse. Supóngase que para ellas el entrenamiento sea severo. No se habla de 'dolor'; especialmente, no en la forma de una conjetura: “quizá él tiene...". Si alguien se queja, se le ridiculiza o se le castiga. Ni remotamente existe la sospecha de simulación. Quejarse es ya, por así decirlo, una simulación" (ibid., 706): "Lo que es interesante para nosotros podría no serlo para ellos. Alli otros conceptos dejarian de ser inconcebibles. En efecto, conceptos esencialmente distintos solo en estas condiciones son concebibles. $(Z, 388)$ " (ibid., 708).

55 "No puedo saber nunca lo que ocurre en el otro; él lo sabe siempre" A quien piense filosóficamente le gustaría decir esto. Pero ¿a qué situación corresponde este enunciado? Oímos a diario que alguien dice sin asomo alguno de duda, refiriéndose a otra persona, que tiene dolores, que está triste, alegre, etcétera; y es más bien raro que oigamos que alguien no sabe lo que está 
"formas de vida" diferentes puede originarse una "duda irremediable": "Tenemos aquí, entonces, lo que quisiera llamar el caso de la duda irremediable. Cuando digo: 'No tengo idea de lo que él está pensando'. Él me resulta un libro cerrado. El único medio para entender a otra persona es haber tenido la misma educación que ella -lo que es imposible-... Pero imagina personas cuya educación se encuentre orientada a suprimir las expresiones de emoción en su rostro y en sus gestos, y supón que esta gente se haga inaccesible para mí pensando en voz alta en un lenguaje que no entiendo. Ahora digo: 'No tengo idea de lo que está ocurriendo en ellas'. Y, sin embargo, allí está: un hecho externo"56 57.

Entonces, es perfectamente posible que una persona puede ser para otra como "un libro cerrado": no es comprensible para ella. Sin embargo, en principio, alguien podría leer un libro cerrado, aunque no mientras permanezca cerrado (en ese sentido una persona puede compararse con un libro, pero no con una silla, por ejemplo). Lo que se quiere decir es que el pensamiento de otra persona no es inaccesible porque esté oculto en su mente ${ }^{58}$, porque posea un carácter privado, sino porque su forma de vida es diferente a la nuestra, y en consecuencia, sus expresiones, también públicas, verbales y no verbales, no resultan descifrables para nosotros. Estamos ante esas personas como lo estamos ante un "hecho externo". Entonces: "Aquellos casos en los que lo interno parece ocultárseme son muy especiales. La inseguridad ${ }^{59}$ que se expresa de esta manera, no es una inseguridad filosófica, sino práctica y primitiva"60.

ocurriendo en otra persona. La incertidumbre no es, entonces, algo tan malo. Y también se dice: "Sé que en aquel momento te sentías así, aunque ahora no lo quieras reconocer" (ibid., 138); "En la conducta misma hay confianza y desconfianza. Si alguien, por ejemplo, se queja, puedo reaccionar con toda seguridad confiadamente, o reaccionar con inseguridad, como alguien que sospecha algo. No se requiere para ello ni de palabras ni de pensamientos" (ibid., 662) (Z 573); "Dada una misma evidencia, alguien puede estar completamente convencido, mientras que otra persona puede no estarlo. Debido a ello, no excluimos a ninguno de los dos de la sociedad como alguien irresponsable o incapaz de hacer un juicio" (ibid., 685). Por otra parte, la incertidumbre no es el fruto del supuesto carácter privado u oculto de los fenómenos mentales: "La incertidumbre no se basa en el hecho de que esa persona no lleve su dolor en la manga" (ibid., 621).

56 Ibid., 568, cursivas de la autora.

57 En el mismo sentido: "La incertidumbre principal. Si él mismo no lo expresa, no sé lo que está 300 pensando. Pero imagina que él lo expresara, pero que lo hiciera en un lenguaje que no entiendes. Podría golpetear con un dedo de una mano en la palma de la otra usando el alfabeto morse o algo parecido. Después de todo, en tal caso resulta también secreto. ¿Y no es tan secreto como si nunca hubiera sido expresado? El lenguaje podría también ser un lenguaje que yo no pudiera aprender nunca; tener, por ejemplo, reglas extremadamente complejas" (ibid., 563).

58 "Alguien puede, por lo tanto, ocultarme sus pensamientos al expresarlos en un lenguaje que no entiendo. Pero ¿dónde está aquí lo mental oculto?” (ibid., 564).

59 Aquí Wittgenstein emplea el mismo concepto, Unsicherheit, que en los otros fragmentos citados se traduce por "incertidumbre" y aquí por "inseguridad".

60 Ibid., 558.

ARETÉ Revista de Filosofía, vol. XXVIII, N², 2016 / ISSN 1016-913X 
Despejado entonces el supuesto teoricista acerca del aprendizaje del lenguaje y de la comprensión psicológica y semántica ordinaria a través de la identificación de formas más básicas (incluso pre-verbales y no verbales) de comprensión reciproca, en las que no hay que transitar de la evidencia preintencional a la comprensión intencional (o del enigma a la teoría), es posible ver mejor no solo de qué modo las certidumbres prácticas son el asiento de nuestras primeras maneras de entendernos, sino también, y correlativamente, que cuando las formas de vida son diferentes, pueden haberse originado (algunos o muchos) conceptos y comportamientos diferentes a los nuestros. En estos casos, nuestras dificultades para entender a los otros son, también, en lo básico, de naturaleza práctica. Solo podemos registrar sus comportamientos, pero no entenderlos, solo podemos observarlos pero no interactuar con ellos. Quizá también traducir con dificultad su lenguaje, pero con recaudos hacia esas diferencias, sin proyectarles nuestras formas parroquiales de conceptualizar.

En efecto, podemos observar y describir lo que otros hacen, porque se trata de hechos de carácter público, pero nos falta la capacidad para usar sus conceptos, para hacer con ellos lo que ellos hacen. Esta situación "radical" solo admite un observador externo, ya no un intérprete: "Uno podría imaginar, por supuesto, que en un filme se le mostrara la vida de los hombres, o que solo se le permitiera observarla, sin tomar parte en ella. Alguien a quien le ocurriera esto entendería la vida humana a la manera como nosotros entendemos la vida de los peces o incluso de las plantas"61.

Si el relativismo conceptual es (al menos) la tesis según la cual hay en principio una diversidad de esquemas conceptuales con los que es posible categorizar la realidad ${ }^{62}$, entonces Wittgenstein admite claramente esta posibilidad. Más aun, en sus escritos se sugiere que no se trata solamente de una posibilidad. Por otra parte, esta tesis no requiere contraponer esquema conceptual y contenido no interpretado (como lo hace Davidson en su crítica al relativismo conceptual ${ }^{63}$ ),

\footnotetext{
61 Ibid., 29.

62 Este es uno de los sentidos del concepto de relatividad conceptual según Bar-On, en "Language, Concepts and Culture: Between Pluralism and Relativism”. Glock, por su parte, distingue entre el relativismo conceptual así definido (atribuible a Wittgenstein) y otras tesis diferentes, como la inconmensurabilidad epistémica y la inconmensurabilidad semántica (Glock, H.-J., "Relativism, Commensurability and Translatability”, en: Preston, J. (ed.), Wittgenstein and Reason, Oxford: Blackwell Publishing, 2008, pp. 21-46).

${ }^{63}$ Glock, en "Relativism Commensurability and Translatibity", analiza el argumento davidsoniano contra el relativismo conceptual basado en la tesis de la diversidad de esquemas conceptuales, mostrando que su conclusión no es correcta, porque sus otras premisas relativas a la intraducibilidad de los lenguajes basados en esquemas conceptuales diferentes, no es correcta. No desarrollo aquí este argumento anti-davidsoniano.
} 
ya que "las diferencias conceptuales y, con ellas, la distancia conceptual, viene en diferentes clases y grados"64: tanto en lo que se refiere a la realidad, cuanto en lo que se refiere a los conceptos, no es necesario ser kantianos. Contra Kant, los rasgos del mundo, humano y no humano imponen algunas constricciones a la formación de conceptos ${ }^{65}$, y esta es la razón por la cual el mundo humano no presenta solo casos de uniformidad o innovación conceptual completas. Siendo así, no se requieren tampoco remedios davidsonianos.

Wittgenstein afirma, además, que no hay criterios objetivos para establecer cuál esquema conceptual es el correcto, puesto que la diferencia conceptual es anterior a su aplicación en juicios y que solo los juicios (o las acciones) pueden ser correctos o incorrectos. Afirmar la relatividad de los criterios de corrección a un esquema conceptual, sin embargo, no disuelve la noción de criterios de corrección (una conclusión que Wittgenstein rechazaría), sino que solo la enmarca. En otras palabras, no solo debe reconocerse como un hecho la diversidad de esquemas conceptuales, sino que debe reconocerse que estos son relativos a una cultura o a una forma de vida en un determinado período histórico, pero que, en ellos o para ellos, sí es posible distinguir entre juicios correctos, falsos o carentes de sentido. El grado de esta diversidad o divergencia conceptual puede reconocerse en el grado de incertidumbre para establecer relaciones prácticas con aquellos a quienes se intenta comprender, pero no tiene sentido afirmar que quienes difieren de nosotros encierran un enigma oculto y suscitan, por lo tanto, dudas filosóficas radicales. Por eso mismo, no se sale de esta dificultad forzando artificiosamente las cosas: afirmando que todos concebimos las cosas del mismo modo (por tener la misma teoría para tratar con los mismos conceptos) o bien que entender a otros nos obliga a proyectarles nuestros modos de entender las cosas, maximizando el acuerdo con ellos. Ni el universalismo ni el parroquialismo son las alternativas correctas para tratar con el problema. Más bien tendriamos que aceptar, como un hecho más de la vida (un hecho acerca del carácter de los conceptos), que hay (y más ampliamente aun, que son posibles) casos de genuina diversidad conceptual:

64 Bar-On, D., "Language, Concepts and Culture: Between Pluralism and Relativism", p. 23. 65 Aunque no me referido este aspecto aquí, Wittgenstein señala en diversos lugares la conexión entre nuestros conceptos y ciertos eventos regulares, familiares, al imaginar de qué modo un cambio abrupto y radical haría inútiles nuestros conceptos o se "trastornarian todos nuestros juicios". Dicho de otra forma: "...si las cosas se comportaran como nunca lo han hecho, tal vez diría algo parecido a: 'Me he vuelto loco'. Pero esto no sería sino una expresión de que he abandonado el intento de ubicarme en relación con las cosas..." El interés de imaginar situaciones así es advertir que "...entre un estado de este tipo y el estado normal no hay una línea de demarcación clara" (Z 393) (OFP, v. II, 605).

ARETÉ Revista de Filosofía, vol. XXVIII, N², 2016 / ISSN 1016-913X 
"Pero esa persona ¿no pasa por alto algo que está presente allí? -No le presta ninguna atención. ¿Y por qué tendría que prestársela? -Justamente en tal caso su concepto es fundamentalmente diferente del nuestro. - ¿Fundamentalmente diferente? Diferente. -Pero entonces, con toda seguridad es como si su palabra no pudiera designar lo mismo que la nuestra. O como si solo designara parte de ello. -Pero así es, por supuesto, como debe presentarse si su concepto es diferente...."66 (OFP, v. II, 640).

Aunque sin dudas el enfoque wittgensteineano aquí delineado se libra de soluciones teóricas para reconstruir nuestros modos de comprensión de significados y de comportamientos intencionales, desde los más básicos que involucran el aprendizaje del lenguaje hasta los más exóticos que exigen un esfuerzo de "interpretación radical", no lo hace al precio de simplificar la caracterización de los factores, efectos y límites que intervienen en cada tipo de caso. Aunque "deja todo como está", nuestra comprensión del carácter de los fenómenos involucrados gana en perspicuidad, refinamiento y complejidad. Y cuando se trata de comprender cómo comprendemos o no comprendemos a otros, esas virtudes no parecen secundarias.

\section{Bibliografia:}

Bar-On, D., "Semantic Eliminativism, and the 'Theory'. Theory of Linguistic Meaning", en: Canadian Journal of Philosophy, Supplementary, v. XXX, 2004, pp. 159-198. http://dx.doi.org/10.1080/00455091.2004.10717604

Bar-On, D., "Language, Concepts and Culture: Between Pluralism and Relativism", en: Facta Philosophica, v. VI (2004), pp. 183-221.

Chomsky, N., Reflections on Language, Nueva York: Pantheon, 1975.

Churchland, P., "Eliminative Materialism and Propositional Attitudes", en: Journal of Philosophy, v. LXXVIII, 2 (1981), pp. 67-90. http://dx.doi.org/10.2307/2025900

Davidson, D., "De la idea misma de un esquema conceptual", en: De la Verdad y de la Interpretación, Barcelona: Gedisa, 1974/1990, pp. 189-203.

Davidson, D., “A Nice Derangement of Epitaphs", en: Truth, Language, and History, Oxford: Clarendon Press, 1986/2005, pp. 89-107. http://dx.doi. org/10.1093/019823757x.003.0007

Davidson, D., "La aparición del pensamiento", en: Subjetivo, Intersubjetivo, Objetivo, Madrid: Cátedra, 1997, pp. 176-190.

Davidson, D., "Meaning, Truth and Evidence", en: Barret, R. y R. Gibson (eds.), Perspectives on Quine, Cambridge Mass.: Blackwell, 1990, pp. 68-79.

Dennett, D., Contenido y Conciencia, Barcelona: Gedisa, 1969/1996.

${ }^{66}$ Ibid., 640.

ARETÉ Revista de Filosofía, vol. XXVIII, Nº 2, 2016 / ISSN 1016-913X 
Fodor, J., The Language of Thought, Cambridge Mass.: Harvard University Press, 1975.

Follesdal, D., "Indeterminacy and Mental States", en: Barret, R. y R. Gibson (eds.), Perspectives on Quine, Cambridge Mass.: Blackwell, 1990, pp. 98-109.

Glock, H.-J., "On Safari with Wittgenstein, Quine and Davidson", en: Arrington, R. y H.J. Glock (eds.), Wittgenstein and Quine, Londres/ Nueva York: Routledge, 1996, pp. 144-172.

Glock, H.-J., "Wittgenstein on Concepts”, en: Ahmed, A. (ed.), Wittgenstein's Philosophical Investigations: a critical guide, Cambridge Mass.: Cambridge University Press, 2010, pp. 88-108. http://dx.doi.org/10.1017/cbo9780511750939.006

Glock, H.-J., "Relativism, Commensurability and Translatability", en: Preston, J. (ed.), Wittgenstein and Reason, Oxford: Blackwell, 2008, pp. 21-46. http://dx.doi. org/10.1002/9781444307092.ch2

Quine, W.O., Palabra y Objeto, Barcelona: Herder, 1960/2001.

Ramberg, B., Donald Davidson's Philosophy of Language: An Introduction, Oxford: Basil Blackwell, 1989.

Sellars, W., "Empirismo y la Filosofia de la Mente", en: Ciencia, Percepción y Realidad, Madrid: Tecnos, 1956/1971, pp. 139-209.

Wittgenstein, L., Investigaciones Filosóficas, México: Crítica, 1998.

Wittgenstein, L., Observaciones sobre los Fundamentos de la Psicología, 2 vv., México: Crítica, 1997.

Wittgenstein, L., Zettel, México: Crítica, 1985.

Wittgenstein, L., "Observaciones sobre La Rama Dorada de Frazer", en: Ocasiones filosóficas. 1912-1951, Madrid: Cátedra, 1997, pp. 144-163.

Recibido: 07/09/15

Aceptado: 19/10/15 